\title{
DISCOVERY OF NEW DWARF GALAXY NEAR THE ISOLATED SPIRAL GALAXY NGC 6503
}

\author{
Jin Koda $^{1}$, Masafumi Yagi ${ }^{2}$, Yutaka Komiyama ${ }^{2}$, Samuel Boissier ${ }^{3}$, Alessandro Boselli ${ }^{3}$, AleXandre Y. K. Bouquin ${ }^{4}$, \\ Jennifer Donovan Meyer ${ }^{5}$, Armando Gil de Paz ${ }^{4}$, Masatoshi Imanishi ${ }^{6}$, Barry F. Madore ${ }^{7}$, and David A. Thilker ${ }^{8}$ \\ ${ }^{1}$ Department of Physics and Astronomy, Stony Brook University, Stony Brook, NY 11794-3800, USA; jin.koda@ stonybrook.edu \\ ${ }^{2}$ Optical and Infrared Astronomy Division, National Astronomical Observatory of Japan, 2-21-1 Osawa, Mitaka, Tokyo 181-8588, Japan \\ ${ }^{3}$ Aix Marseille Université, CNRS, LAM (Laboratoire d'Astrophysique de Marseille) UMR 7326, F-13388 Marseille, France \\ ${ }^{4}$ Departamento de Astrofísica y CC. de la Atmósfera, Universidad Complutense de Madrid, Avda. de la Complutense s/n, E-28040 Madrid, Spain \\ ${ }^{5}$ National Radio Astronomy Observatory, 520 Edgemont Road, Charlottesville, VA 22903, USA \\ ${ }^{6}$ Subaru Telescope, 650 North A'ohoku Place, Hilo, HI 96720, USA \\ ${ }^{7}$ Carnegie Institution of Washington, 813 Santa Barbara Street, Pasadena, CA 91101, USA \\ ${ }^{8}$ Center for Astrophysical Sciences, The Johns Hopkins University, 3400 N. Charles Street, Baltimore, MD 21218, USA \\ Received 2015 February 17; accepted 2015 March 11; published 2015 April 2
}

\begin{abstract}
We report the discovery of a new dwarf galaxy (NGC 6503-d1) during the Subaru extended ultraviolet disk survey. It is a likely companion of the spiral galaxy NGC 6503. The resolved images, in the $B, V, R, i$, and $\mathrm{H} \alpha$ bands, show an irregular appearance due to bright stars with underlying, smooth and unresolved stellar emission. It is classified as the transition type (dwarf irregular (dIrr)/dwarf spheroidal $(\mathrm{dSph})$ ) between the dIrr and $\mathrm{dSph}$ types. Its structural properties are similar to those of the dwarfs in the Local Group, with an absolute magnitude $M_{V} \sim-10.5$, half-light radius $r_{e} \sim 400 \mathrm{pc}$, and central surface brightness $\mu_{0, V} \sim 25.2$. Despite the low stellar surface brightness environment, one $\mathrm{H}$ II region was detected, though its $\mathrm{H} \alpha$ luminosity is low, indicating an absence of any appreciable O-stars at the current epoch. The presence of multiple stellar populations is indicated by the color-magnitude diagram of $\sim 300$ bright resolved stars and the total colors of the dwarf, with the majority of its total stellar mass $\sim 4 \times 10^{6} M_{\odot}$ in an old stellar population.
\end{abstract}

Key words: galaxies: dwarf - galaxies: individual (NGC 6503) - galaxies: irregular - galaxies: stellar content

\section{INTRODUCTION}

Dwarf satellite galaxies provide vital guidance for understanding star formation (SF) and galaxy evolution, and their discovery and structural analysis are of broad interest. They often have extremely low surface brightness (LSB) and exhibit rare instances of SF in the low-density environments. They are potential triggers of SF in their more prominent parent galaxies. The observed statistics and internal structures of dwarf satellites in the Local Group are challenging the $\Lambda \mathrm{CDM}$ scenario (Klypin et al. 1999; Moore et al. 1999; BoylanKolchin et al. 2012). Systematic searches for dwarf satellites around other galaxies, including a more rigorous one in the Local Group, are being undertaken (e.g., McConnachie 2012; Monachesi et al. 2014; Merritt et al. 2014; Sand et al. 2014; Tollerud et al. 2015; McQuinn et al. 2015; Donovan Meyer et al. 2014).

In this Letter, we report the discovery of a new dwarf galaxy NGC 6503-d1, approximately $17^{\prime}$ away from the spiral galaxy NGC 6503. NGC 6503 is comparatively isolated at the edge of the Local Void (Karachentsev et al. 2003; Greisen et al. 2009). It is an $\mathrm{SA}(\mathrm{s}) \mathrm{cd}$ type spiral galaxy at a distance of $d=5.27 \pm 0.53 \mathrm{Mpc} \quad\left(1^{\prime \prime}=25.5 \mathrm{pc} ; \quad\right.$ Karachentsev et al. 2003) with a heliocentric velocity of $v_{h}=25 \pm 1 \mathrm{~km} \mathrm{~s}^{-1}$ (Epinat et al. 2008). NGC 6503 has a major axis of $D_{25} \sim 10.9 \mathrm{kpc}(7 ! 1)$ and an absolute magnitude of $M_{V}=-19.07$ (calculated from de Vaucouleurs et al. 1991). Assuming a mass-to-light ratio $\gamma_{V} \sim 1.2$ (see McGaugh \& Schombert 2014), NGC 6503's stellar mass is $\sim 4 \times 10^{9} M_{\odot}$, and its virial radius is approximately $150 \mathrm{kpc}$ (see Kravtsov 2013). We present the basic properties, stellar populations, and recent SF activity of the new dwarf galaxy NGC 6503-d1. Uncertainty still remains as to the distance of NGC 6503-d1, but based on its proximity and other supporting evidence the dwarf is most likely associated with NGC 6503 .

NGC 6503 shows very extended ultraviolet (XUV) emission far beyond the edge of its optical disk (Thilker et al. 2007). Such XUV disks indicate SF at a very low density (Koda et al. 2012) and are quite common (residing in $30 \%$ of nearby galaxies; Thilker et al. 2007). However, the trigger of the XUV activity still remains unknown (e.g., Espada et al. 2011). NGC 6503-d1 is the first dwarf satellite discovered during the early observations of the Subaru-XUV survey, a deep optical imaging survey of nearby XUV disks using the Subaru telescope.

\section{OBSERVATIONS AND DATA REDUCTION}

The dwarf galaxy was discovered in deep imaging of the spiral galaxy NGC 6503 using the the Subaru Prime Focus Camera (Suprime-Cam; Miyazaki et al. 2002) on the Subaru telescope. Suprime-Cam has a mosaic of ten $2048 \times 4096$ CCDs, covering a wide field of $34^{\prime} \times 27^{\prime}$ with a pixel scale of $0 ! \prime 20$. The observations were performed in 2013 June and July and supplemented with archival data taken in 2012 July. The field of NGC 6503 was observed in the $B, V, R, i$, and NA656 $(\mathrm{H} \alpha)$ bands, among which the images in $V$ and $i$ were deeper. The typical seeing was 0.8 .

The data were reduced in a standard way, including an overscan correction, masking of the shadow of the autoguider probe, flat-fielding, and distortion correction before co-adding all exposure frames (Yagi et al. 2002). Photometric calibrations were performed using a catalog from the Panoramic Survey Telescope \& Rapid Response System 1 (Pan-STARRS 1; Schlafly et al. 2012; Tonry et al. 2012; Magnier et al. 2013). We followed the procedure described in Yagi et al. (2013) and converted from the Pan-STARRS 1 to the Subaru band system 

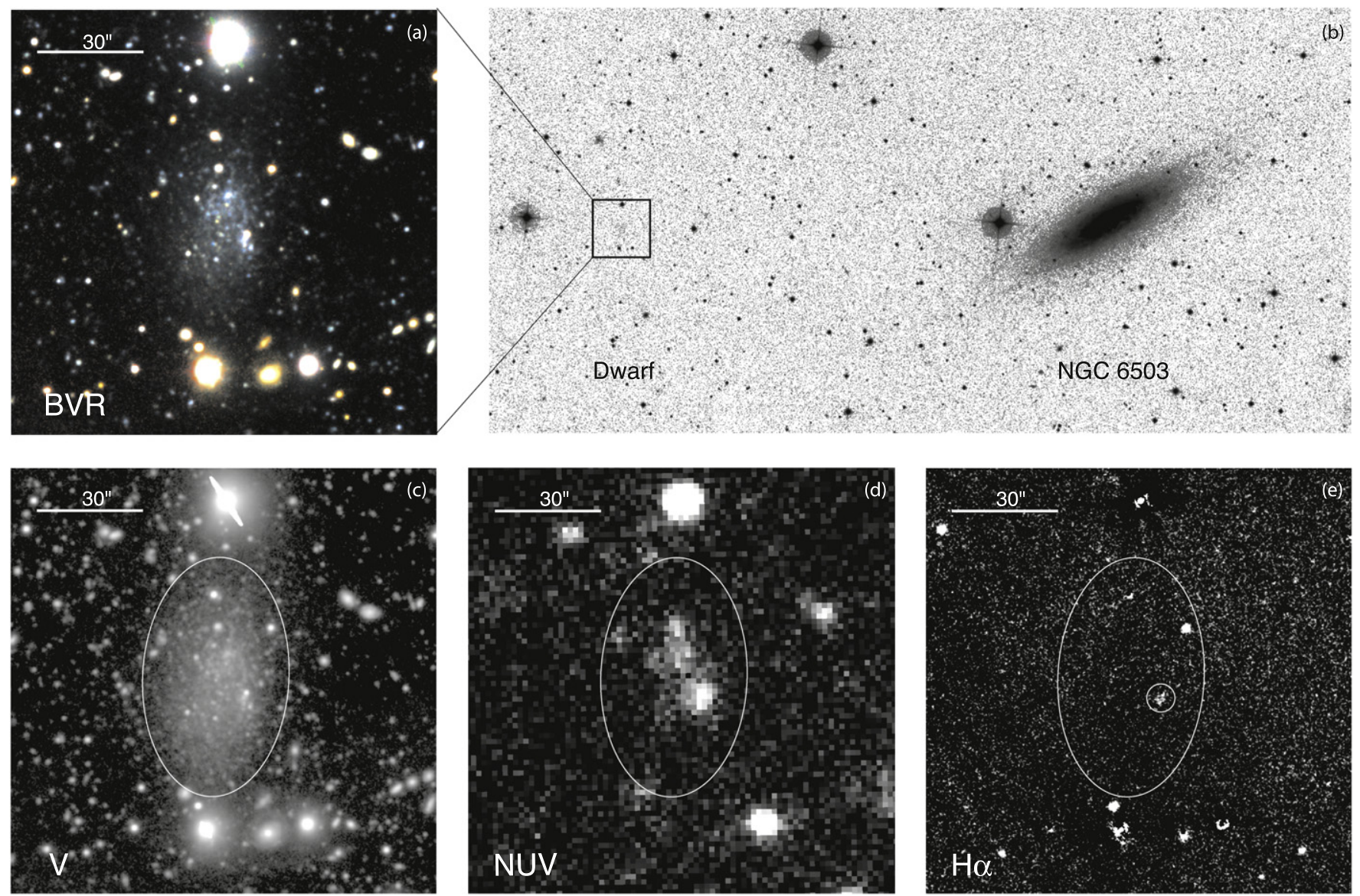

Figure 1. Subaru images of $2^{\prime} \times 2^{\prime}$ region around the dwarf, except at top right, which is a $\sim 30 \prime 2 \times 12$.7 region from the Digitized Sky Survey (DSS). North is up, and east is left. The photometric aperture is an ellipse with a major axis of $\sim 68^{\prime \prime}$, axis ratio of 0.61 , and position angle of -2.6 from north to east. (a) Pseudo-color image with Subaru $B, V$, and $R$. (b) DSS image of NGC 6503 and the new dwarf galaxy. (c) Subaru $V$-band image in log scale. (d) GALEX NUV image. (e) Subaru $\mathrm{H} \alpha$ image $($ net $\mathrm{H} \alpha)$. The small circle encloses the identified $\mathrm{H}$ II region.

using stellar photometry. Typical photometric errors including both statistical and systematic effects are $\lesssim 0.1 \mathrm{mag}$.

We also obtained archival FUV and NUV images from the $G A L E X$. We averaged two available data sets from the GALEX Nearby Galaxies Survey (Gil de Paz et al. 2007) and from the General Investigator Program (GI1 Program 097).

\section{DISCOVERY AND CLASSIFICATION}

Figure 1 shows the new dwarf galaxy NGC 6503-d1. The $B V R$ color image (panel a) shows a lopsided distribution of resolved bright stars in the northern part of the dwarf, apparently indicating an irregular morphology. However, we also found a smooth, extended, and symmetric stellar distribution in logarithmic scale (panel c; $V$-band example), which is perhaps a more accurate representation of the stellar mass distribution. Panel (e) shows the continuum-subtracted $\mathrm{H} \alpha$ image. $\mathrm{H} \alpha$ emission is detected at one spot within the dwarf. The presence of the resolved bright stars and the $\mathrm{H}_{\text {II }}$ region indicate that this dwarf hosts recent/current SF and, by implication, some gas, though no $\mathrm{HI}$ gas was detected at the $3 \sigma$ upper limit of $<10^{6} M_{\odot}$ (Greisen et al. 2009).

We classify this as a transition-type dwarf spheroidal $(\mathrm{dSph}) / \mathrm{dwarf}$ irregular (dIrr), based on traditional definitions (Tolstoy et al. 2009; McConnachie 2012), the type between $\mathrm{dIrr}$ and $\mathrm{dSph}$. We note, however, that there is a discussion on whether dIrrs and dSphs are physically distinct, the only difference being their current/recent SF which outshines the other populations and gives the impression of an irregular morphology (e.g., Weisz et al. 2011).

\section{PROPERTIES}

NGC 6503-d1 is very likely a satellite galaxy of NGC 6503. Their projected separation $\sim 17^{\prime}$ is well inside NGC 6503's virial radius $\sim 100^{\prime}(\sim 150 \mathrm{kpc})$, suggesting a high probability of their physical association $(>80 \%)$ based on $N$-body simulations (e.g., Tollerud et al. 2011). The $\mathrm{H} \alpha$ detection also supports its proximity to NGC 6503, though the heliocentric velocity of NGC $6503\left(v_{h}=25 \pm 1 \mathrm{~km} \mathrm{~s}^{-1}\right)$ is very close to MW's and it is difficult to distinguish between NGC 6503 and the MW. Although uncertainty still remains, we assume that the dwarf is a companion of NGC 6503 in what follows. We will discuss a few pieces of supporting evidence in addition to the proximity, including its size and magnitude (distance-dependent parameters) compared to other dwarf galaxies (Section 4.2) and a likely detection of the tip of red giant branch (TRGB) star population (Section 4.3). Each of these alone is insufficient to prove that the dwarf is a satellite of NGC 6503. The combination, however, supports our working assumption, that $d=5.25 \mathrm{Mpc}$ is the distance to NGC 6503-d1. 
Table 1

Properties of NGC 6503-D1

\begin{tabular}{|c|c|}
\hline Parameter & Value \\
\hline \multicolumn{2}{|c|}{ Distance-independent Properties } \\
\hline R.A. (J2000) & $17: 52: 48.4$ \\
\hline Decl. (J2000) & $+70: 08: 14.4$ \\
\hline Galactic longitude $l$ (degree) & 100.5403 \\
\hline Galactic latitude $b$ (degree) & 30.3563 \\
\hline$V_{h}\left(\mathrm{~km} \mathrm{~s}^{-1}\right)$ & $\sim 0$ \\
\hline FUV (AB mag) & 21.1 \\
\hline NUV (AB mag) & 20.4 \\
\hline$B(\mathrm{AB} \mathrm{mag})$ & 18.5 \\
\hline$V(\mathrm{AB} \mathrm{mag})$ & 18.1 \\
\hline$R(\mathrm{AB} \mathrm{mag})$ & 17.8 \\
\hline$i(\mathrm{AB} \mathrm{mag})$ & 17.6 \\
\hline Sérsic index $n$ & 0.54 \\
\hline$\mu_{0, V}\left(\mathrm{AB} \operatorname{mag} \operatorname{arcsec}^{-2}\right)$ & 25.2 \\
\hline$r_{e}(")$ & 15.6 \\
\hline Axis ratio & 0.61 \\
\hline P.A. $(\operatorname{deg}, \mathrm{N}$ to $\mathrm{E})$ & -2.6 \\
\hline
\end{tabular}

Distance-dependent Properties

\begin{tabular}{lc}
\hline$m-M(\mathrm{mag})$ & $28.61 \pm 0.23$ \\
$d(\mathrm{Mpc})$ & $5.27 \pm 0.53$ \\
$M_{\mathrm{FUV}}(\mathrm{AB} \mathrm{mag})$ & -7.6 \\
$M_{\mathrm{NUV}}(\mathrm{AB} \mathrm{mag})$ & -8.2 \\
$M_{B}(\mathrm{AB} \mathrm{mag})$ & -10.1 \\
$M_{V}(\mathrm{AB} \mathrm{mag})$ & -10.5 \\
$M_{R}(\mathrm{AB} \mathrm{mag})$ & -10.8 \\
$M_{i}(\mathrm{AB} \mathrm{mag})$ & -11.0 \\
$r_{e}(\mathrm{pc})$ & 400 \\
$F_{H}\left(\mathrm{erg} \mathrm{s}{ }^{-1}\right)$ & $8.2 \times 10^{35}$ \\
Stellar mass $\left(M_{\odot}\right)$ & $\sim 4 \times 10^{6}$ \\
$\mathrm{HI}$ gas mass $\left(M_{\odot}\right)$ & $<10^{6}$ \\
\end{tabular}

Notes. All magnitudes are corrected for Galactic extinction. Typical errors due to photometric calibration and background contamination are $\sim 0.14$ mag.

\subsection{Internal Structure}

The GALFIT package (Peng et al. 2002) was adopted to measure structural parameters of the dwarf. The fit was made to the $V$-band image with a Sersic profile (Sérsic 1968) and fixed sky background after removing some surrounding stars. We obtained a Sérsic index of $n=0.54$ with $\chi_{\nu}^{2}=2.8$ $(\nu=90,494)$. This result was consistent with that using the $i$-band image. As noted earlier, some bright stars within the dwarf outshine the underlying smooth stellar distribution. After aggressively masking these we obtained $n=0.63\left(\chi_{\nu}^{2}=0.63\right)$ though it is debatable whether the member stars of the dwarf should be masked out. Table 1 gives properties of NGC 6503$\mathrm{d} 1$, including its central coordinates, axis ratio, position angle (P.A.), and effective radius/half-light radius $r_{e}$, as well as other global parameters. The central surface brightness $\mu_{V}$ was derived from the value at the central pixel of the fitted model, and the result after Galactic extinction correction, $\mu_{V}=25.18 \mathrm{mag} \operatorname{arcsec}^{-2}$, is typical for dwarf galaxies (McConnachie 2012).

Apparent magnitudes (AB magnitude) were calculated within an elliptical aperture (Figure 1). The shape of the aperture was derived from GALFIT (i.e., an axis ratio and P. A.) with the major axis diameter scaled to $34^{\prime \prime}$ so as to enclose the emission of the dwarf. The fluxes of foreground/background contamination were estimated by placing the apertures in the surrounding region. We subtracted their averages from the fluxes within the dwarf aperture and adopted their typical scatters of $0.1 \mathrm{mag}$ as errors in photometry. The apparent magnitudes are listed in Table 1. We adopted a Galactic extinction of $A_{V}=0.106$ (Schlegel et al. 1998; Schlafly \& Finkbeiner 2011) and calculated extinctions in UV and NA656 with $\left(A_{\mathrm{FUV}}, A_{\mathrm{NUV}}\right)=(2.58,2.83) A_{V}$ for the Milky Way (Koda et al. 2012) and $A_{\mathrm{NA} 656}=A_{R}$. These aperture magnitudes were consistent with the ones from GALFIT (e.g., 18.2 mag in $V$ ).

\subsection{Statistical Properties}

If it is a satellite of NGC 6503, NGC 6503-d1 lies within the sequence of dwarf galaxies in the Local Group, in surface brightness $\mu_{V}$, absolute magnitude $M_{V}$, and half-light radius $r_{e}$. Figure 2 shows their correlations with Local Group dwarfs (McConnachie 2012) and Galactic globular clusters (Harris 1996) as references. The new dwarf is distinct from the globular clusters and lies midway between dIrrs (blue) and dSphs (yellow) and among the dIrr/dSph transition types (green). This location is consistent with the visual appearance of the dwarf, having apparent irregular morphology (due to recent SF) with the more symmetric morphology of the underlying stellar population.

The Sérsic profile of $n=0.54$ is also within the range typical of dwarf galaxies of similar brightness. Radial profiles of dwarfs are often fit with an exponential profile (an $n=1$ profile; e.g., Ibata et al. 2007; Richardson et al. 2011; Crnojević et al. 2014), though $n=0.3-0.7$ has been reported for dwarf galaxies around M101 (Merritt et al. 2014) and an isolated dwarf galaxy (Monachesi et al. 2014). Geha et al. (2006) showed a spread of $n \sim 0.5-1.5$, albeit among brighter dwarfs ( $\lesssim-14 \mathrm{mag}$ ). The structural properties of the new dwarf are, therefore, typical of faint dwarf galaxies in the Local Group and in some other nearby systems.

\subsection{Resolved Stars}

The high spatial resolution of the Subaru images $(\sim 0$ "! 8$)$ permits identification and photometry of individual bright stars within NGC 6503-d1. We made an initial catalog of 312 potential detections using the DAOPHOT PSF photometry software (Stetson 1987). We then manually rejected apparent misidentifications (e.g., background galaxies and tails between adjacent bright stars), leaving 303 stars, which are identified in Figure 3(a). We performed photometry in $V$ and $i$ bands, made a color-magnitude diagram (CMD; middle) and a histogram of apparent $i$ magnitude for stars with $V-i>0.6$ (right).

Theoretical isochrones are superimposed on the CMD assuming a distance of NGC $6503(m-M=28.62 \pm 0.23)$ and were taken from the Padova and Trieste stellar evolutionary tracks (Bressan et al. 2012; Chen et al. 2014; Tang et al. 2014). ${ }^{9}$ The red isochrones are at an age of $10 \mathrm{Gyr}$ for metallicities of $\log \left(\mathrm{Z} / \mathrm{Z}_{\odot}\right)=-2.0,-1.5,-1.0$, and -0.5 from the left to right. The others are for $\log \left(\mathrm{Z} / \mathrm{Z}_{\odot}\right)=-0.5$ and at $\log ($ Age $[$ year] $)=7-10$ (see legend). The bright stars that we identified are around the areas of the asymptotic giant branch (AGB), horizontal branch, and red giant branch (RGB) sequences. Therefore, the dwarf hosts multiple stellar

\footnotetext{
http://stev.oapd.inaf.it/cgi-bin/cmd
} 


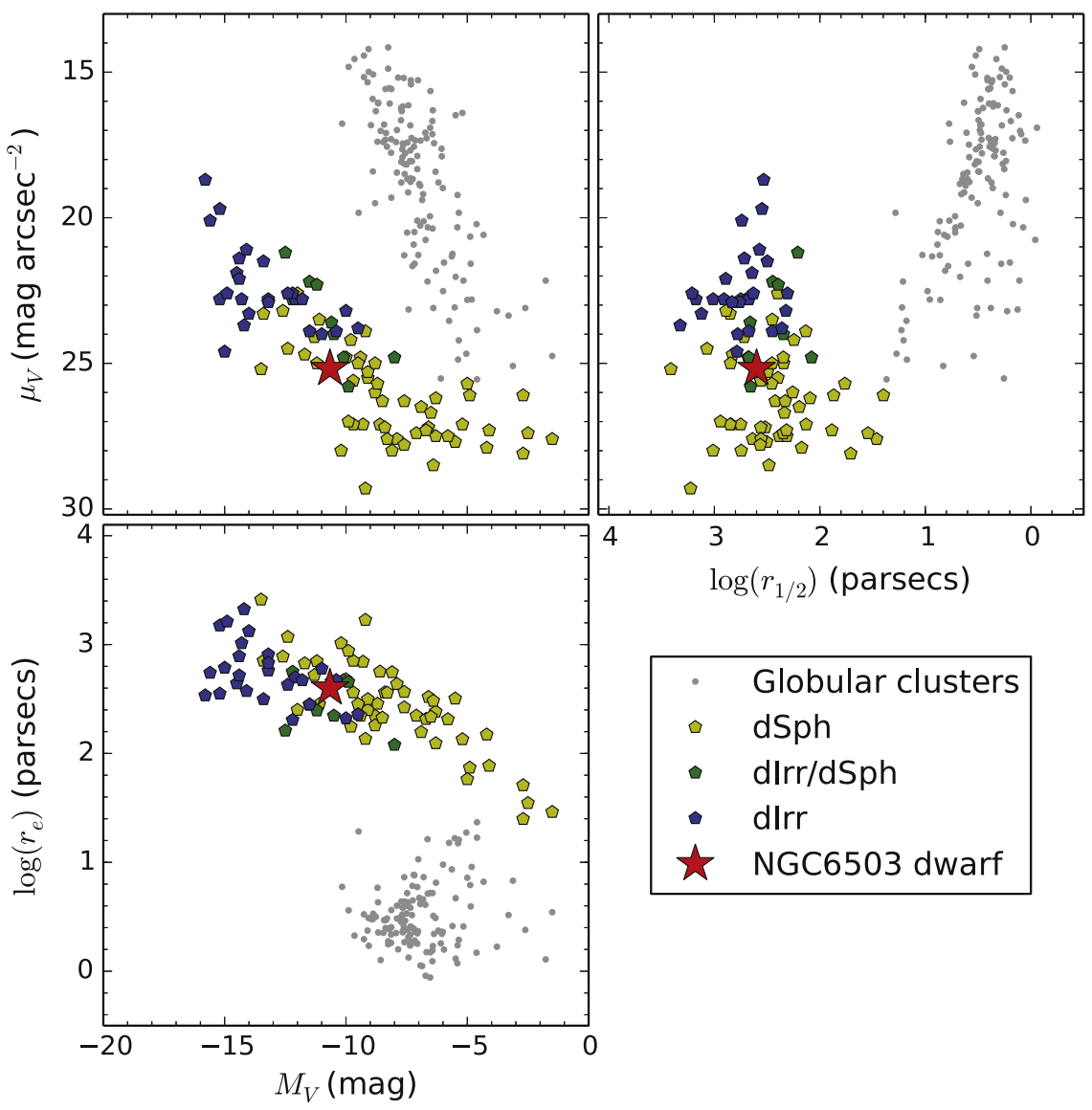

Figure 2. Correlations between central surface brightness $\mu_{V}$, half-light radius $r_{e}$ and absolute $V$ magnitude $M_{V}$. The red star is the new dwarf galaxy. Comparison data are dwarf spheroidal (dSph), dwarf irregular (dIrr), and transition (dIrr/dSph) galaxies in the Local Group compiled by McConnachie (2012) and Galactic globular clusters from Harris (1996). We adopted the distance of NGC 6503 for NGC 6503-d1.

populations, from young ( $100 \mathrm{Myr}$, cyan isochrone) to old ( $\gtrsim 8-10$ Gyr, magenta and red). Many stars in the recently formed population must have escaped their natal dust given the agreement between unreddened isochrones and our photometry, especially regarding the blue limit of the He-coreburning population. The RGB sequence depends on stellar metallicity (red lines; see also Lee et al. 1993). The spread on the red side may indicate a variety of metallicity in the underlying stellar population.

The tip of RGB (TRGB) stars are often used as a distance indicator, as their luminosities are approximately constant near the $i$-band wavelength (Lee et al. 1993). By averaging the four red isochrones in Figure 3(b) we determined a TRGB magnitude of $M_{i}=-3.53 \mathrm{AB}$ mag in the Subaru $i$ band. If the dwarf is a companion of NGC 6503, the corresponding apparent magnitude is $m_{i}=25.09$ with an error of at least \pm 0.23 carried over from the distance modulus. Within the errors, this is roughly where we see the rise in the histogram (Figure 3(c); $V-i>0.6$ for TRGB color), though the sample is small. This supports the assumption that the dwarf is a companion of NGC 6503.

\subsection{SF History}

The central and average $V$-band surface brightnesses are 25.18 and $26.31 \mathrm{mag} \operatorname{arcsec}^{-2}$, respectively, after correcting for the Galactic extinction. Any SF there is a rare incident in a very LSB regime. Conventionally, galaxies sizes have been defined by the $25 \mathrm{mag} \operatorname{arcsec}^{-2}$ isophote level. The distribution of resolved stars in the CMD suggests multiple stellar populations, indicating that $\mathrm{SF}$ has persisted for a long period of time. Figure 1(e) shows the detection of one $\mathrm{H}_{\text {II }}$ region (small circle) within the dwarf at $6 \sigma$ significance. The $\mathrm{H}$ II region is virtually co-incident with the brightest spot in NUV (GALEX J175247.4+700811; Figure 1(d)), indicating current $\mathrm{SF}$ in this LSB galaxy. [The brightest $\mathrm{H} \alpha$ spot at NW without NUV emission is unlikely to be in the dwarf, since it would be too bright.]

The SF activity of this $\mathrm{H}_{\text {II }}$ region is low, which is inferred from its faint $\mathrm{H} \alpha$ luminosity of $L_{\mathrm{H}_{\alpha}}=8.2 \times 10^{35} \mathrm{erg} \mathrm{s}^{-1}$. This was measured within an $8^{\prime \prime}$-diameter aperture centered at $\left(\alpha_{\mathrm{J} 2000}, \delta_{\mathrm{J} 2000}\right)=(17: 52: 47.1,+70: 08: 09.8)$ with correction for Galactic extinction. The uncertainty is about a factor of 2 due to a sensitivity to continuum subtraction (The fraction of $\mathrm{H} \alpha$ over total emission in the NA656 band is $~ 5 \%$ in the aperture). The contamination of $[\mathrm{N} \mathrm{II}] \lambda$ 6548, 6583 emission is likely negligible (see Kennicutt et al. 2008). This $L_{\mathrm{H}_{\alpha}}$ is on the order of that expected around a single B0V type star $\left(\sim 7.0 \times 10^{35} \mathrm{erg} \mathrm{s}^{-1}\right.$ under case $\mathrm{B}$ recombination with an electron temperature of $10^{4} \mathrm{~K}$; Sternberg et al. 2003; Osterbrock \& Ferland 2006; Koda et al. 2012). This is consistent with observations of the Orion Nebula in the Milky Way, which is about 10 times brighter in $L_{H_{\alpha}}$ with only a single O5.5 V type star as a dominant ionizing source. The second brightest stars in Orion (one $09.5 \mathrm{~V}$, two B0.5 V, and one B3; 

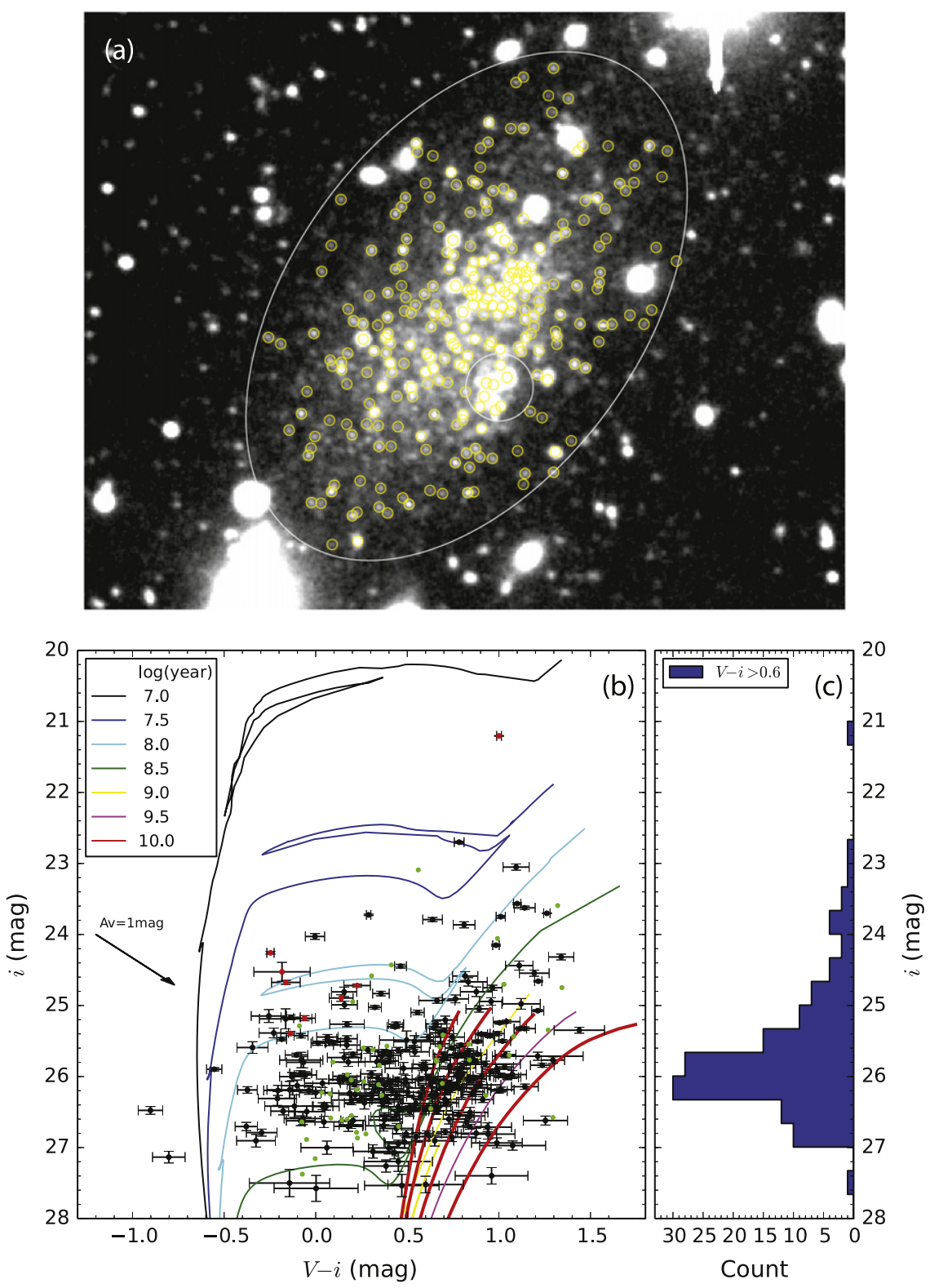

Figure 3. Analysis of resolved stars. (a) Locations of resolved stars (yellow circles) in the $i$-band image. The image orientation is not rotated from the original to maintain the high spatial resolution. The white ellipse and circle (H II region) are the same as the ones in Figure 1. (b) Color-magnitude diagram (CMD) with the $V$ and $i$ bands. The stellar isochrones are from the Padova evolutionary tracks $(\mathrm{CMD} 2.7)$ and of $\log \left(\mathrm{Z} / \mathrm{Z}_{\odot}\right)=-0.5$ at $\log (\mathrm{Age})=7.0,7.5,8.0,8.5,9.0,9.5$, and 10.0. The red isochrones are of $\log (\mathrm{Age})=10.0$ for $\log \left(\mathrm{Z} / \mathrm{Z}_{\odot}\right)=-2.0,-1.5,-1.0$, and -0.5 from left to right, respectively. We applied the distance modulus of $\mathrm{NGC} 6503$ $(m-M=28.62 \pm 0.23)$. Stars within the HII region aperture (white circle in panel (a)) are marked red. For comparison, the green dots are objects in a control field of the same size about $2^{\prime}$ away from NGC 6503-d1 (at the same distance from NGC 6503); these background objects are populated much more sparsely in the CMD (this is so, even though the difference in confusion limit is not corrected). (c) Histogram of apparent $i$-band magnitude for all identified stars of $V-i>0.6$. The increment is $0.33 \mathrm{mag}$. From the bright to faint, the number starts increasing between the bins of $m_{i}=25.17$ and 25.50. The AB magnitude of TRGB in Subaru $i$ is approximately -3.53 (absolute) and $25.09 \pm 0.23$ (apparent; see the text).

Reipurth 2008) together emit 10-20 times fewer Lyman continuum photons (e.g., Sternberg et al. 2003). Therefore, the dwarf galaxy is unlikely to be hosting any appreciable O-star population at the current time, assuming that the leakage of ionizing photons is not dominant $(\gtrsim 90 \%$ of the Lyman continuum photons need to leak away without ionizing the surrounding gas to hide an O-star). Of course, it could have had O-star(s) a short time ago as their lifetimes are short $(\sim 10 \mathrm{Myr})$. The stars around this $\mathrm{H}_{\text {II }}$ region are AGB and blue loop stars (Figure 3). One, or some, of these may be the ionizing source if an internal extinction is locally large $\left(A_{V} \sim 1\right)$.
The observed colors of the dwarf (Table 1) also indicate multiple epochs of SF. Rigorous analyses of SF history (e.g., Weisz et al. 2011, 2012) are beyond our scope, and a dwarf galaxy of small mass requires stochasticity in SF to be taken into account (e.g., da Silva et al. 2012). Instead, we adopt a simplistic approach to illustrate that at least two stellar populations, old and young, are necessary to account for the observed colors, and that most of the stellar mass is old. We adopted Starburst99 (Leitherer et al. 1999), a Kroupa initial mass function, a metallicity of $Z=0.4 Z_{\odot}$, and an internal extinction $A_{V}=0.1$ for the integrated colors of the dwarf. These choices were made arbitrarily for demonstration; for 

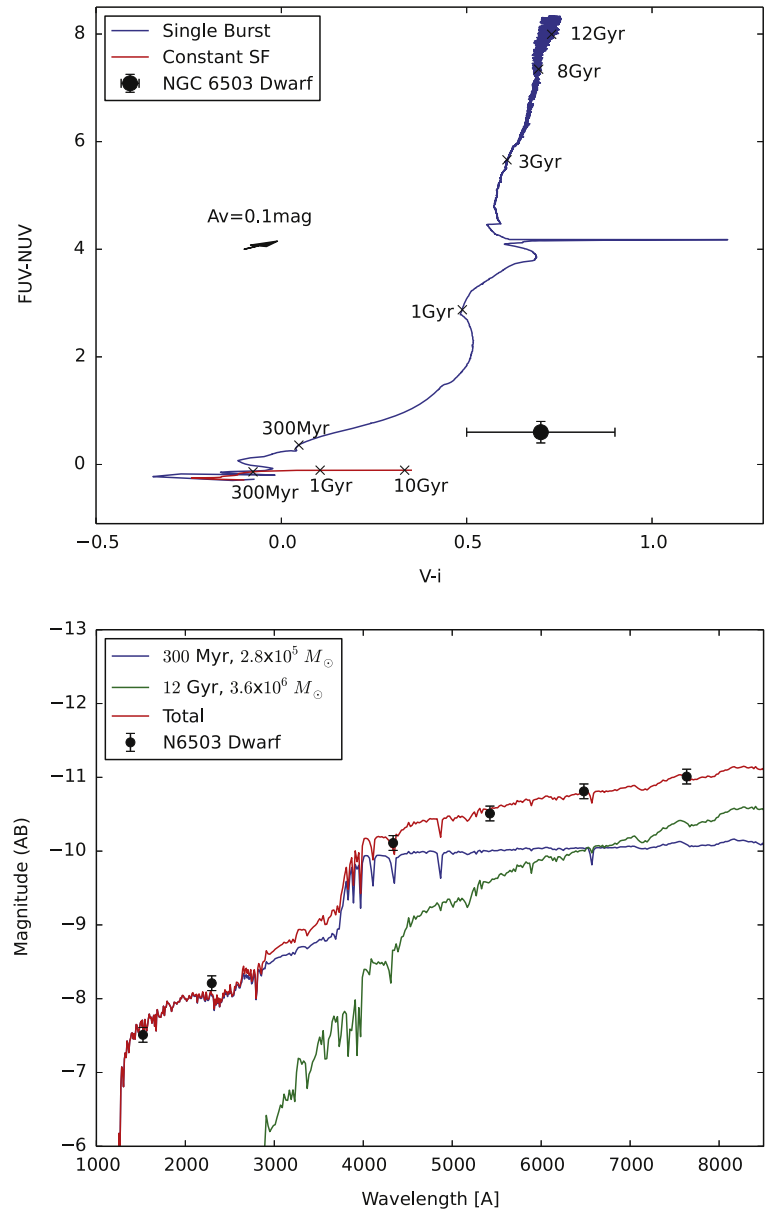

Figure 4. Comparisons of photometric evolution models (Starburst99) and the data for the dwarf. A metallicity of $0.4 Z_{\odot}$ and a Kroupa initial mass function are adopted. (a) FUV-NUV vs. V-i plot. A single burst model (blue) and constant SF model (red) are plotted. The arrow shows an internal extinction of $A_{V}=0.1 \mathrm{mag}$ using the extinction curve of LMC (Pei 1992), which is not applied to the data nor models. (b) Spectra from the single burst model at ages $=300 \mathrm{Myr}$ (blue) and $12 \mathrm{Gyr}$ (green), and the summation of the two (red). A correction for internal extinction $\left(A_{V}=0.1\right)$ was applied. The zeropoints of the spectra (i.e., stellar masses) were adjusted by eye to approximately fit the observed FUV, NUV, $B, V, R$, and $i$ magnitudes of the dwarf.

example, the metallicity may have a range from a low $\log \left(Z / Z_{\odot}\right)$ to $>-0.5$ according to Figure 3 (the data with respect to the RGB isochrones).

Figure 4(a) shows that neither a model of single starburst at any age nor continuous SF alone reproduces the FUV-NUV and $\mathrm{V}-\mathrm{i}$ colors simultaneously unless $A_{V}$ is unusually high. Figure 4(b) shows an example of stellar populations at the young and old ages of $300 \mathrm{Myr}$ and $12 \mathrm{Gyr}$. The young population (blue) is relatively bright in FUV and NUV and shows a flat spectrum in the redder, optical range $(B, V, R$, and $i$ ). The old population (green) contributes negligibly in the FUV and NUV and shows a rising spectrum in the optical bands. The dwarf is relatively bright in FUV and NUV (requiring a young stellar population) and shows a rising trend in the optical bands (old stellar population). The masses of stellar populations determine the zero point in magnitudes: the old component carries the most mass $\left(\sim 3.6 \times 10^{6} M_{\odot}\right.$; insensitive to the actual age if $\gtrsim 8 \mathrm{Gyr}$ ) as compared to the young one $\left(\sim 2.8 \times 10^{5} M_{\odot}\right)$. The total stellar mass within the dwarf is approximately $4 \times 10^{6} M_{\odot}$.

\section{SUMMARY}

We have discovered the new dwarf galaxy of the dIrr/ Sph transition type, NGC 6503-d1, which is likely a physical companion of the spiral galaxy NGC 6503. Its structural properties are similar to those of the Local Group dwarfs. Integrated colors and resolved stars indicate a complex SF history not described by a single burst of any particular age nor by a simple continuous SF, which is also similar to the other known dwarfs (Weisz et al. 2011, 2012). The majority of the total stellar mass $\sim 4 \times 10^{6} M_{\odot}$ is in an old stellar population. NGC 6503-d1 is the only companion galaxy of this magnitude near NGC 6503 within our field of view, about one-sixth of the virial radius. It is possible, and likely, that more dwarfs can be found in a future survey.

This research used data collected with the Subaru Telescope operated by the National Astronomical Observatory of Japan and with the GALEX operated for NASA by the Caltech under NASA contract NAS5-98034, and used the NASA/IPAC Extragalactic Database (NED) operated by the Jet Propulsion Laboratory, Caltech, under contract with NASA. We thank an anonymous referee for constructive comments, Fumiaki Nakata for help in observations, Eric Greisen for HI data, and the PanSTARRS1 Surveys collaboration for the photometric catalog. This research is supported by NASA through grant NNX14AF74G. J.K. also acknowledges support from the NSF (AST-1211680).

\section{REFERENCES}

Boylan-Kolchin, M., Bullock, J. S., \& Kaplinghat, M. 2012, MNRAS, 422, 1203

Bressan, A., Marigo, P., Girardi, L., et al. 2012, MNRAS, 427, 127

Chen, Y., Girardi, L., Bressan, A., et al. 2014, MNRAS, 444, 2525

Crnojević, D., Sand, D. J., Caldwell, N., et al. 2014, ApJL, 795, L35

da Silva, R. L., Fumagalli, M., \& Krumholz, M. 2012, ApJ, 745, 145

de Vaucouleurs, G., de Vaucouleurs, A., Corwin, H. G., Jr., et al. 1991, Third

Reference Catalogue of Bright Galaxies (New York: Springer)

Donovan Meyer, J., Peek, J. E. G., Putnam, M., \& Grcevich, J. 2014, ApJ, submitted

Epinat, B., Amram, P., Marcelin, M., et al. 2008, MNRAS, 388, 500

Espada, D., Muñoz-Mateos, J. C., Gil de Paz, A., et al. 2011, ApJ, 736, 20

Geha, M., Blanton, M. R., Masjedi, M., \& West, A. A. 2006, ApJ, 653, 240

Gil de Paz, A., Boissier, S., Madore, B. F., et al. 2007, ApJS, 173, 185

Greisen, E. W., Spekkens, K., \& van Moorsel, G. A. 2009, AJ, 137, 4718

Harris, W. E. 1996, AJ, 112, 1487

Ibata, R., Martin, N. F., Irwin, M., et al. 2007, ApJ, 671, 1591

Karachentsev, I. D., Makarov, D. I., Sharina, M. E., et al. 2003, A\&A, 398, 479

Kennicutt, R. C., Jr, Lee, J. C., Funes, J. G., et al. 2008, ApJS, 178, 247

Klypin, A., Kravtsov, A. V., Valenzuela, O., \& Prada, F. 1999, ApJ, 522, 82

Koda, J., Yagi, M., Boissier, S., et al. 2012, ApJ, 749, 20

Kravtsov, A. V. 2013, ApJL, 764, L31

Lee, M. G., Freedman, W. L., \& Madore, B. F. 1993, ApJ, 417, 553

Leitherer, C., Schaerer, D., Goldader, J. D., et al. 1999, ApJS, 123, 3

Magnier, E. A., Schlafly, E., Finkbeiner, D., et al. 2013, ApJS, 205, 20

McConnachie, A. W. 2012, AJ, 144, 4

McGaugh, S. S., \& Schombert, J. M. 2014, AJ, 148, 77

McQuinn, K. B. W., Cannon, J. M., Dolphin, A. E., et al. 2015, arXiv: 1501.07313

Merritt, A., van Dokkum, P., \& Abraham, R. 2014, ApJL, 787, L37

Miyazaki, S., Komiyama, Y., Sekiguchi, M., et al. 2002, PASJ, 54, 833

Monachesi, A., Bell, E. F., Radburn-Smith, D. J., et al. 2014, ApJ, 780, 179

Moore, B., Ghigna, S., Governato, F., et al. 1999, ApJL, 524, L19

Osterbrock, D. E., \& Ferland, G. J. 2006, Astrophysics of Gaseous Nebulae and Active Galactic Nuclei (Sausalito, CA: University Science Books) 
Pei, Y. C. 1992, ApJ, 395, 130

Peng, C. Y., Ho, L. C., Impey, C. D., \& Rix, H.-W. 2002, AJ, 124, 266

Reipurth, B. 2008, in Handbook of Star Forming Regions, Volume I: The Northern Sky, ed. B. Reipurth (San Francisco, CA: ASP)

Richardson, J. C., Irwin, M. J., McConnachie, A. W., et al. 2011, ApJ, 732, 76

Sand, D. J., Crnojević, D., Strader, J., et al. 2014, ApJL, 793, L7

Schlafly, E. F., \& Finkbeiner, D. P. 2011, ApJ, 737, 103

Schlafly, E. F., Finkbeiner, D. P., Jurić, M., et al. 2012, ApJ, 756, 158

Schlegel, D. J., Finkbeiner, D. P., \& Davis, M. 1998, ApJ, 500, 525

Sérsic, J. L. 1968, Atlas de Galaxias Australes (Córdoba, Argentina: Observatorio Astronómico)

Sternberg, A., Hoffmann, T. L., \& Pauldrach, A. W. A. 2003, ApJ, 599,1333
Stetson, P. B. 1987, PASP, 99, 191

Tang, J., Bressan, A., Rosenfield, P., et al. 2014, MNRAS, 445, 4287

Thilker, D. A., Bianchi, L., Meurer, G., et al. 2007, ApJS, 173, 538

Tollerud, E. J., Boylan-Kolchin, M., Barton, E. J., Bullock, J. S., \& Trinh, C. Q. 2011, ApJ, 738, 102

Tollerud, E. J., Geha, M. C., Grcevich, J., Putman, M. E., \& Stern, D. 2015, ApJL, 798, L21

Tolstoy, E., Hill, V., \& Tosi, M. 2009, ARA\&A, 47, 371

Tonry, J. L., Stubbs, C. W., Lykke, K. R., et al. 2012, ApJ, 750, 99

Weisz, D. R., Dalcanton, J. J., Williams, B. F., et al. 2011, ApJ, 739, 5

Weisz, D. R., Johnson, B. D., Johnson, L. C., et al. 2012, ApJ, 744, 44

Yagi, M., Kashikawa, N., Sekiguchi, M., et al. 2002, AJ, 123, 66

Yagi, M., Suzuki, N., Yamanoi, H., et al. 2013, PASJ, 65, 22 